\title{
Experimental Modelling of Propulsion Transients of a Brushless DC Motor and Propeller Pair under Limited Power Conditions: A Neural Network Based Approach
}

\author{
Muhammed T. Köroğlu*. Mert Önkol.* \\ Mehmet Önder Efe* \\ *Dept. of Electrical and Electronics Engineering, TOBB Economics and Technology University, Söğ̈̈tözü, Ankara \\ Turkey (Tel: +90-312-292 4064; e-mail: onderefe@etu.edu.tr).
}

\begin{abstract}
This paper presents an experimental framework to describe the dynamic behavior of brushless direct current (BLDC) motors, which are frequently used in unmanned aerial vehicle (UAV) applications. Typically these applications require varying the angular speeds of motors which have to be precise parts of the dynamic model. The experimental setup is a motor/propeller pair equipped with an electronic speed controller. The substantial contribution of the paper is to incorporate the voltage drop caused by the consumed power and the alleviation of the modulation effect on the measured battery voltage. The consequence of the voltage drop is to obtain different angular speeds under same excitation inputs. A Neural Network (NN) based approach is chosen to handle this modeling issue. Levenberg-Marquardt algorithm is used to tune the adjustable parameters of $\mathrm{NN}$, which is trained offline using the data observed through a set of experiments. Some experimental validation results are presented to justify the model.
\end{abstract}

\section{INTRODUCTION}

System identification has always been at the center of the feedback control system design (Juang 1994, Ljung 1999). Many techniques have been developed and the devised reliable models have been used for various engineering applications. In some cases, it is possible to collect data from the process yet no prior model structure is available, e.g. difference or differential equations. Such cases are generally difficult to cope with as the data may be expensive, noisy or even non-descriptive. Employing methods exploiting the numerical data become useful in such cases, e.g. as in the case of Narendra and Parthasarathy (1990). That study motivated many researchers and pioneered the use of connectionist structures with suitable adjustment schemes in feedback control systems.

Artificial neural networks with very powerful learning schemes are particularly useful if the process under investigation is described by numerical data. In the literature, several structures are cited frequently, feedforward structure, recurrent structure, radial basis function structure and special structures containing Elman networks are just to name a few, (Haykin, 1994), and among which, feedforward neural network structure has become the most popular as it is able to solve nonlinear regression problems very efficiently. Needless to say, if there are many measurements, many inputs, and tightly coupled interactions between the variables involved, such a structure might be a good choice to explore as reported by (Negnevitsky and Pavlovsky, 2005; Chu et al 1990; Mohamed and Koivo, 2004). One such application example is from the realm of aerial vehicles. Such systems are propulsion based and have a nonlinearities coming from the dynamics as well as the operating environment. A typical setup considers utilizing brushless DC motors, as they have very high torque properties, and electronic periphery with propellers to obtain the necessary forces and torques to maintain the motion (Pounds et al 2006). There are many research outcomes in the literature on brushless DC motor identification. Tipsuvanporn at al (2002) implements the identification of a brushless DC motor and error backpropagation method for adjusting the weights and biases is exploited. Rubaai and Kankam (1997) use dynamic error backpropagation and Kim and Fok (2004) utilize LevenbergMarquardt algorithm to identify a sewing machine. Clearly these works constitute a subset showing the diversity in selecting the parameter update criteria in neural structures. Aside from these, once could choose conjugate gradient and its variants, or reinforcement learning, depending on the nature of the problem in hand (Haykin, 1994).

This paper presents the modelling of propulsion transients and steady values by using a feedforward neural network structure and Levenberg-Marquardt optimization technique. Although this does not seem new, the alleviation of modulated nature of the Lithium Polymer battery voltage and based on its filtered value, the precise estimation of the thrust under the whole range of input values constitute the originality and contribution of this work.

This paper is organized as follows: The second section introduces the test equipment; the third section presents the data acquisition hardware. Next section is devoted to the neural modelling technique, where the implementation results and validation of the model are discussed in detail. The conclusions are given at the end of the paper. 


\section{TEST EQUIPMENT}

\section{1 dSPACE DS1104 R\&D Data Controller Board}

DS1104 is a single-board PCI hardware that combines a data acquisition system with an independent processing system to implement digital control approaches. dSPACE system has breakout panel for connecting signal lines to the DS1104 controller board. Breakout board consists of analog to digital converters (ADCs), digital to analog converters (DACs), serial communication interfaces (rs232, rs422, rs485), pulse width modulation (pwm) outputs, digital incremental encoder interfaces, digital I/O modules enabling the user to work with various types of data captured from real time environment. Operation of the DS1104 board is controlled through

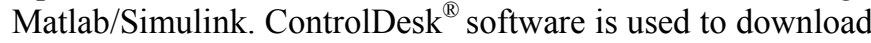
the devised models and approaches to the board, and enables creating layouts for interfacing with variables and parameters of the system.

\subsection{Electronic Speed Controller (ESC)}

Brushless speed controller functions as an interface between the motor and power supply. Controlled by the pwm signal generated by DS1104 board, the brushless ESC provides variable power to the motor allowing proportional speed adjustments. Unlike a brushed motor, brushless motors need special driving periphery to obtain the desired motion. The speed control appropriately powers each phase of the brushless motor in sequence, causing it to get actuated. This work deals with ESC hardware that has three motor wires, allowing it to connect to the standard three phase brushless DC motors.

\subsection{Brushless Direct Current Motor (BLDC Motor)}

BLDC motors have many advantages such as better speed versus torque characteristics, high efficiency, long operating life and noiseless operation when compared to brushed DC motors. Because of high torque characteristics at low speeds, lightweight nature and small sizes, brushless DC motors eliminate the need for gearing mechanisms or large sized motors to obtain certain level of torque values. For the long term goals of the work presented here, AXI 2212/34 outrunner type brushless motor is utilized in the experiments.

\subsection{Propeller}

Peculiar to quadrotor type unmanned vehicles, counter rotating (CR) propeller pairs must be selected to provide inevitably nonnegative- control input which is a distinctive constraint when various dimensions of propellers are considered. For this reason, elimination has been accomplished with different dimensions of $\mathrm{CR}$ propellers considering the pwm signal vs. angular speed characteristics and $12 \times 4.5 \mathrm{CR}$ propeller is chosen, where the length is 12 inches and the pitch is 4.5 inches per revolution.

\subsection{Lithium-Polymer Battery}

Lithium Polymer batteries have become the most preferable electric power source available for UAV research recently. The reason for this is that lithium battery packs are significantly lighter and have higher electrical capacity than its alternatives. These advantages make them ideal for long flight times entailing high currents, which is a necessary condition when the tasks of UAVs are considered. In our experiments, a 3 Cell $2000 \mathrm{mAh}$ Lithium Polymer battery is used to power the ESC.

\subsection{Phototransistor-Infrared Emitter Diode Set-up}

A conventional approach to measure angular speed of a rotating motor is to utilize rotary encoders. In this experimental identification process, a practical way is chosen to capture angular speed data of the BLDC motor/propeller system. A Silicon NPN Phototransistor (BPW76) circuit is placed parallel to an Infrared Emitter Diode (IED) (L51XXIR1BC) circuit. Voltage difference, occurred at collector lead of phototransistor by rotating blades, is transferred to DS1104 hardware and the raw data is recorded. A post-processing algorithm is applied to row data to obtain angular velocity data of the BLDC motor/propeller pair. The details are discussed in the sequel.

\section{DATA ACQUISITION AND ELIMINATING THE MODULATION IN BATTERY VOLTAGE}

\subsection{Voltage of Lithium-Polymer Battery}

Data acquisition hardware captures voltages under $10 \mathrm{~V}$ indicating that a voltage divider is necessary to scale the battery voltage into the measurable range. Voltage data of experiments are read by DS1104 via an ADC Channel. As shown in Fig. 1, the battery voltage is severely modulated due to the electrical interactions in between the driving circuitry. Since the thrust is relevant to the angular speed and angular speed is relevant to the battery conditions, it is of crucial importance the current electrical state of the battery. Clearly the pwm profile given in Fig. 2 causes a decrease in the measured voltage yet there seems a meaningful mean value that could be used for our purposes. The figure describes the central problem addressed here. The digital filter given by (1) is exploited to obtain a useful information quantifying the instant value of the battery voltage.

$$
H(z)=4.9975 \times 10^{-4} \frac{z+1}{z-0.9995}
$$

According to the results in Fig. 1, it is also obvious that when pwm signal is a constant value (see Fig. 2), filtered voltage tends to decrease due to power consumption. A more precise graph is given in Figs. 3-4 showing the decrease of battery voltage under the same pwm level. Higher pwm levels lead to quicker discharge of the energy yet lower pwm levels cause slower discharge pictures. 


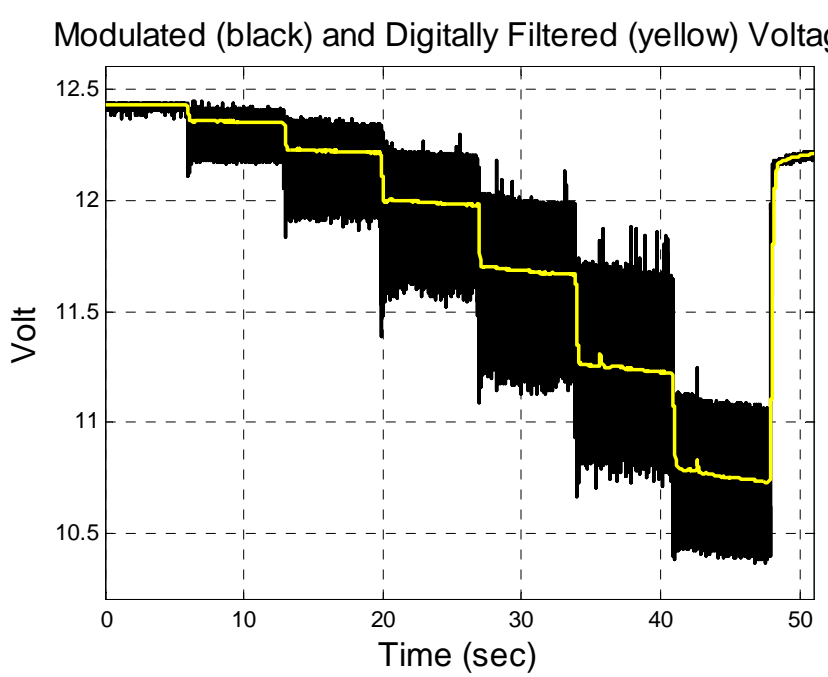

Fig. 1: Voltage of Battery and output of digital filter

Filtered Battery Voltage

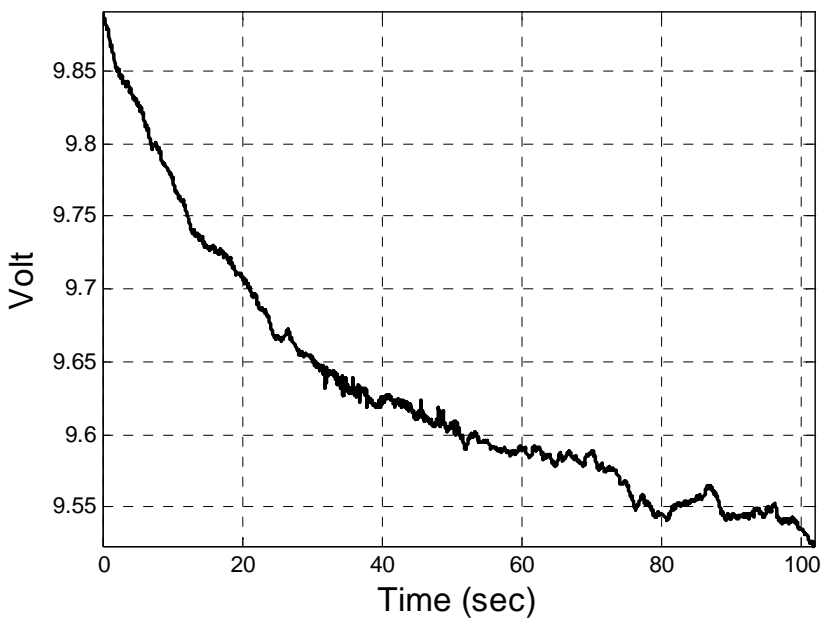

Fig. 3: Battery consumption when pwm level is fixed constant

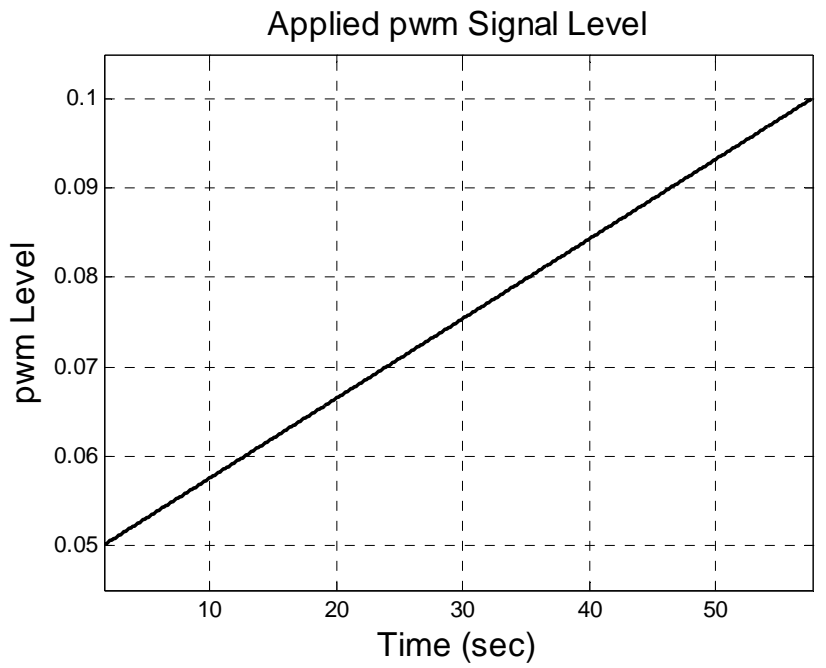

Fig 5: The pwm signal sweeping the whole applicable range

The variation in the battery voltage due to the changing levels of pwm input and the current energy conditions of the battery is a critical issue that needs to be incorporated into the mathematical model with care.

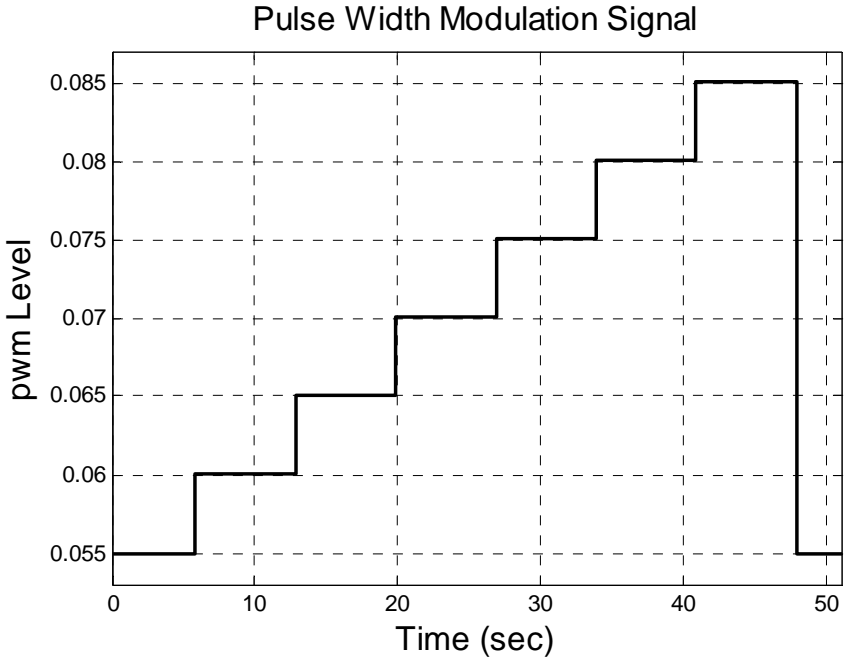

Fig. 2: The applied pwm signal

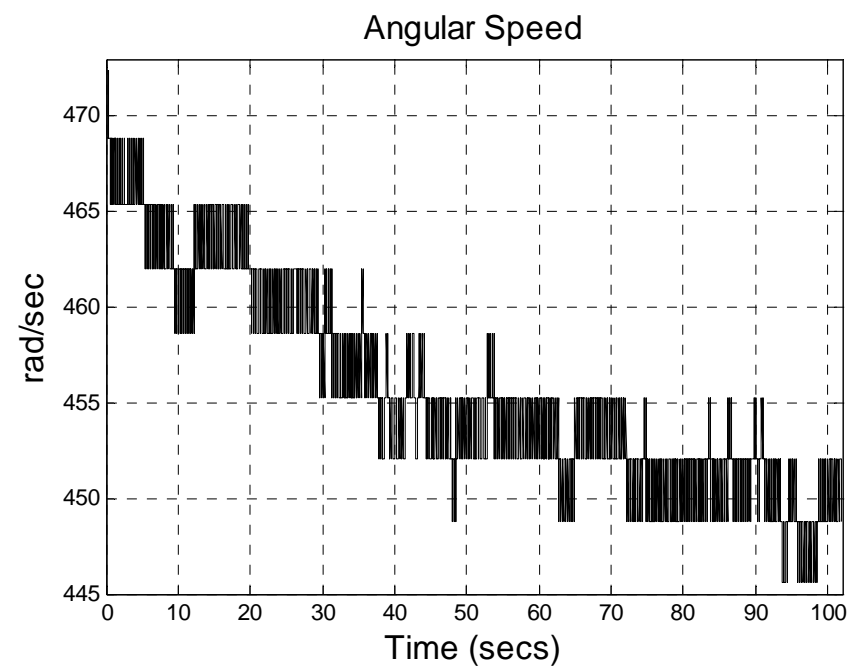

Fig. 4: Change of the angular speed when pwm signal is kept

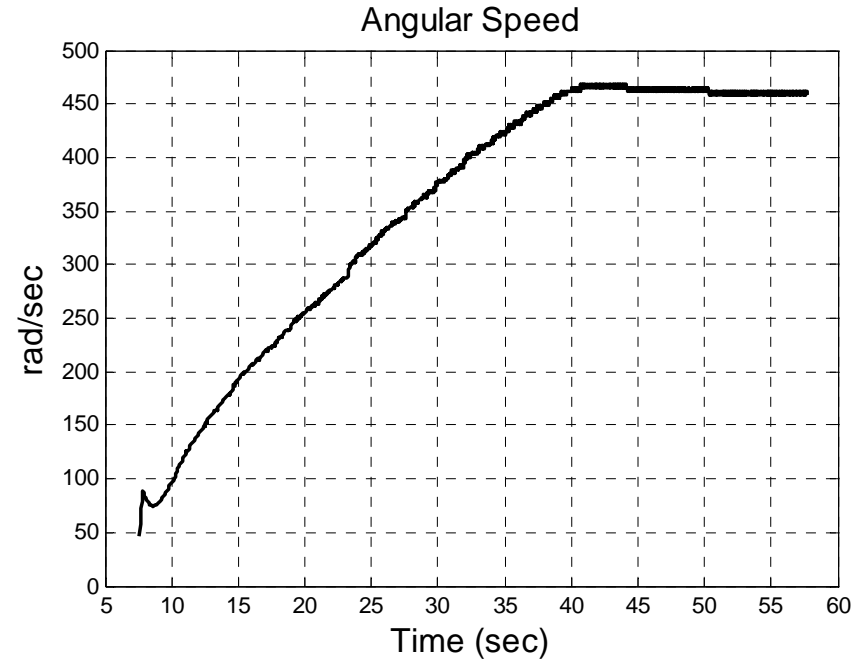

Fig. 6: The corresponding angular speed graph

\subsection{Pulse Width Modulation Signal}

In generating the necessary pulsing to excite the ESC system, regardless of the pwm frequency, duty cycle varies between 
$1 \mathrm{~ms}$ (pwm level is 0.05 ) and $2 \mathrm{~ms}$ (pwm level is 0.10). In Fig. 5 , a pwm profile changing from $1 \mathrm{~ms}$ to $2 \mathrm{~ms}$ linearly is depicted and in Fig. 6, the angular speed observed in response to this pwm profile is shown. The motion starts when pwm level reaches 0.055 and the nonlinearity at the beginning of angular speed graph corresponds to start of rotary motion. In Fig. 7, the driving scheme of AXI 2212/34 outrunner type BLDC motor with ESC is explained.

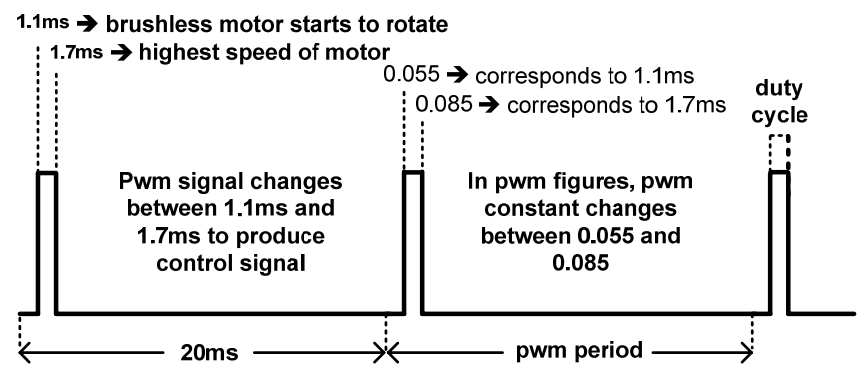

Fig. 7: $50 \mathrm{~Hz}$ pwm signal

\subsection{Angular Speed of BLDC Motor/Propeller}

Since the thrust produced by rotating propeller corresponds to the control signal, the static relationship between the angular speed $w$ and the thrust $f$ should be determined experimentally. This reduces to finding out the $\mathbf{b}$ coefficient that is intrinsic to every particular BLDC motor and propeller pair. The coefficient also depends on aerodynamic circumstances like density of air.

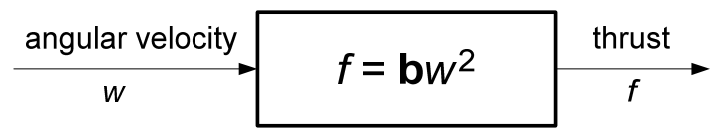

Fig. 8: Analytic relationship between thrust $\&$ angular speed

Phototransistor-IED circuit is prepared to measure angular speed of brushless motor/propeller system, which is significantly cheaper than rotary encoders that may load the motor and cause inexact measurements of angular speeds. To be able to reach the angular speed information utilizing such a simple hardware, the edges of the propeller have been enumerated such that the trailing and leading edges are identified by numbers as shown in Fig. 9. Such a numbering scheme leads to the numbering of rising and falling edges of the signal collected from the circuit shown below.

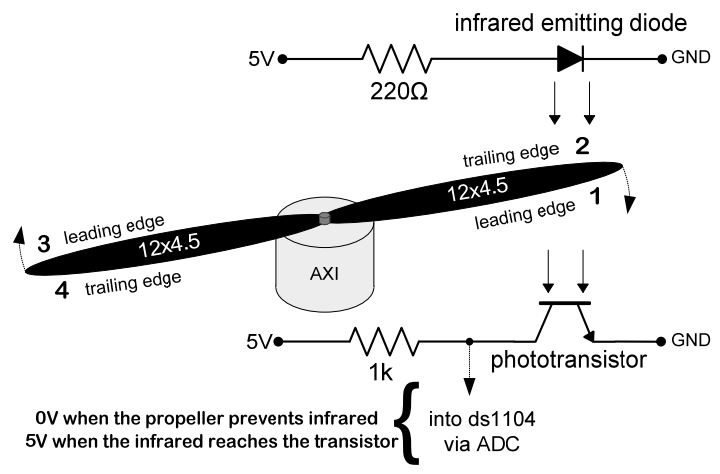

Fig. 9: Phototransistor-IED setup
A typical view of the captured pulses is shown in Fig. 10, where the numbers shown in Fig. 9 and Fig. 10 have same physical meanings. Since the angular speed is a time varying quantity, reading of the pulses provided by the circuit shown in Fig. 9 should be synchronized with a reference time index. Clearly $2 \pi$ radians of turn (one tour) will cause observation of the edges 1,2, 3 and 4, and the fifth edge will form a value of angular speed at that particular instant. After the first value is calculated, each 4 edges of blades preventing and allowing infrared triggering the base terminal of phototransistor creates an angular speed information at that instant of time. Applying a synchronization algorithm to row data recorded in DS1104 via $A D C$, angular speed data is calculated by post-processing in Matlab® environment.

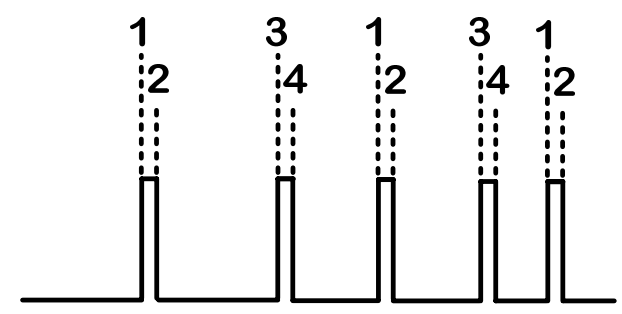

Fig. 10: Pulses generated by rotating propeller blades

\section{NEURAL NETWORK BASED MODELING}

Due to powerful mapping capabilities, neural networks have had a profound effect on the applications of data based modeling applications. This particular property is mainly because of the fact that real systems have many variables, the variables involved in the modeling process are typically noisy, and the underlying physical phenomenon is generally nonlinear in nature. Due to the inextricably intertwined nature of the describing differential (or difference) equations, which are not known precisely, it becomes a tedious task to see the relationship between the variables involved. In such cases, black box models, such as neural networks, fuzzy logic or the methods adapted from the artificial intelligence come into the picture as tools representing the input/output behavior accurately. In what follows, we describe briefly the Levenberg-Marquardt training scheme for adjusting the parameters of a neural structure (Hagan and Menhaj, 1994). Since the algorithm is a soft transition in between the Newton's method and the standard gradient descent, it very quickly locates the global minimum (if achievable) of the cost hypersurface, which is denoted by $J$ in (2).

$$
J=\frac{1}{2} \sum_{p=1}^{P}\left(d_{p}-y_{p}(u, \varphi)\right)^{2}
$$

where $y_{p}$ denotes the response of the single output neural network, $d_{p}$ stands for the corresponding target output. In (2), $\phi$ is the set of all adjustable parameters of the neural structure (weights and the biases), and $u$ is the vector of inputs which are selected according to the following procedure.

In devising the dynamic model of a system by neural models, it is of substantial importance to choose a good set of inputs. For synthetic systems, one simply chooses the inputs and the 
states as the inputs to the neural model, and predicts a function of the state variables. Practically, we know that the required pwm level changes with the battery voltage. Secondly, we utilize the shifted and scaled pwm signal as another input and thirdly, to capture the transients of the thrust, we filter the pwm signal by a first order filter of time constant 0.25 and apply its output as the third input. The output of the neural model is a variable from which the angular speed is obtained. One can of course augment the neural network input with delayed values of these variables yet it is useless to prefer such an approach as our ultimate goal is to utilize this model on a lightweight UAV carrying limited computing facility.

The training architecture of the NN structure is illustrated in Fig. 11 and the adjustment strategy, the LevenbergMarquardt update law is given in (3).

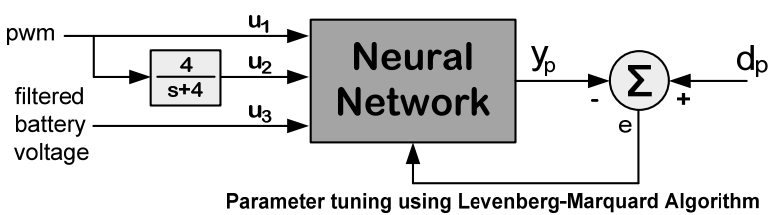

Fig. 11: NN structure

$$
\phi(t+1)=\phi(t)-\left(\mu I+\Psi(t)^{\mathrm{T}} \Psi(t)\right)^{-1} \Psi(t)^{\mathrm{T}} E(t)
$$

where, $\mu$ is the regularization parameter, $E(\mathrm{t})=\left[e_{1} e_{2} \ldots e_{P}\right]^{\mathrm{T}}$ is the vector of errors described as $e_{i}=d_{i}-y_{i}(u, \phi) i=1,2, \ldots, P$, where $P$ is the number of training pairs and $\Psi$ is the Jacobian given explicitly by (4).

$$
\Psi=\left[\begin{array}{cccc}
\frac{\partial e_{1}}{\partial \varphi_{1}} & \frac{\partial e_{1}}{\partial \varphi_{2}} & \cdots & \frac{\partial e_{1}}{\partial \varphi_{N}} \\
\frac{\partial e_{2}}{\partial \varphi_{1}} & \frac{\partial e_{2}}{\partial \varphi_{2}} & \cdots & \frac{\partial e_{2}}{\partial \varphi_{N}} \\
\vdots & \vdots & \ddots & \vdots \\
\frac{\partial e_{P}}{\partial \varphi_{1}} & \frac{\partial e_{P}}{\partial \varphi_{2}} & \cdots & \frac{\partial e_{P}}{\partial \varphi_{N}}
\end{array}\right]
$$

where there are $N$ adjustable parameters within the vector $\phi$. According to the update law given in (3), we carried out several experiments to collect training data. These contain the pwm profiles of staircase and triangular (ramp) structure. During the training, we used 62050 patterns and another 62050 are reserved for testing routine, which stops the training at an appropriate instant beyond which the memorization phenomenon emerges. It should be noted that the training and testing data sets are not identical. The neural model has 3 inputs, 25 hidden neurons and single output, which is linear. The structure is determined experimentally. The hidden nodes have hyperbolic tangent type nonlinear activation functions and before the training starts, the training data and the testing data are scaled and shifted to fit within the range \pm 1 . The evolution of the cost $J$ is depicted in Fig. 12 , where it is seen that the training takes about 250 epochs. The developed model has been checked with a chirp signal shown in Fig. 13. The top left subplot of the figure shows a noisy pwm input of chirp type. This signal was never encountered during the training cycles and it is a likely input signal for a real time flight experiment of a UAV. The filtered battery voltage is depicted in the bottom left subplot, which emphasizes that the filter works perfectly to extract a meaningful value. The top right subplot shows the angular speed predicted by the neural network and the software counting the edges of the propeller experimentally. Finally, the bottom right subplot of the figure illustrates the difference between these two quantities. Clearly, when the frequency of the pwm signal increases, the model performs poorly, conversely, for the slowly changing pwm profiles, the model predicts the angular speed precisely. Considering the physical conditions of a UAV, we conclude that the performance observed by the use of a neural model with described inputs is satisfactory.

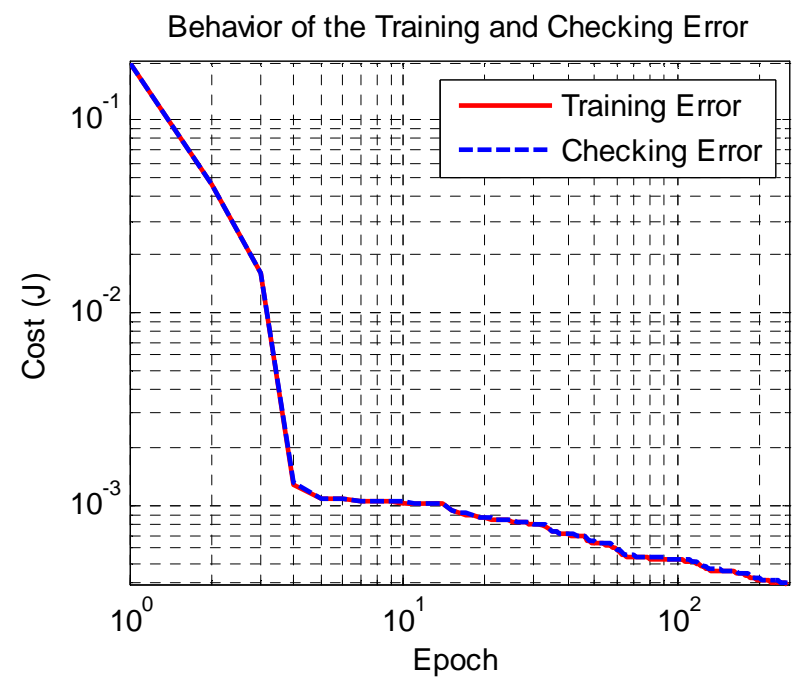

Fig. 12: Decrease of the training and checking errors

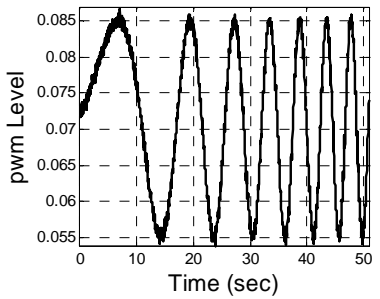

Desired and Predicted Angular Speeds
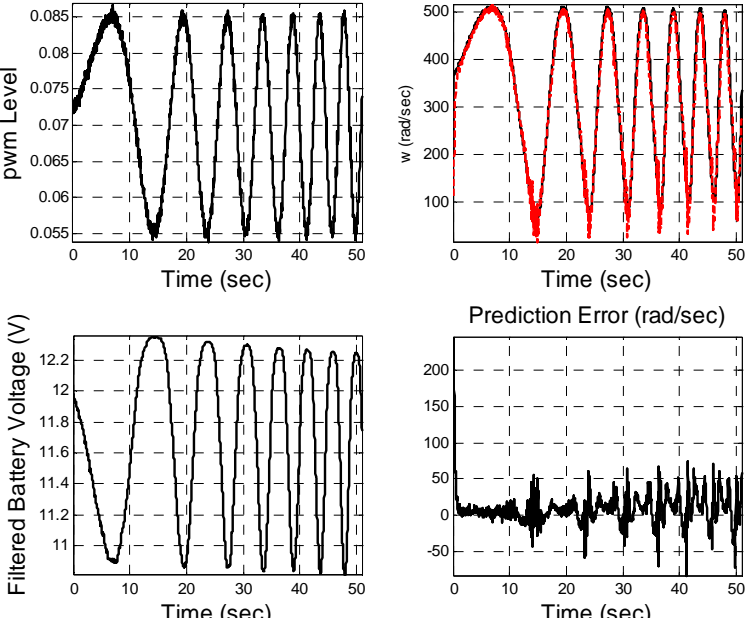

Prediction Error (rad/sec)

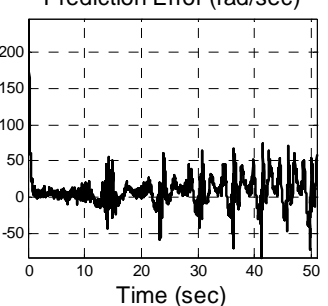

Fig. 13: Experimental results validating the neural model

\section{CONCLUSIONS}

An offline training of neural networks for the identification of a BLDC motor/propeller actuator system has been presented in this paper. Obtained model has been verified for number of test data. Graphical results show that the analytic model is capable of representing the transient and steady vales of the 
propulsion accurately. The critical issue addressed in this paper is the use of battery voltage as a parameter influencing the performance and filtering of the modulation effect. This is accomplished through the use of a digital filter and a simple neural network structure is chosen to discover the relevant map. The results have shown that the neural model performs adequately.

The authors aim at utilizing the mathematical model obtained here for command and control of a quadrotor type UAV.

\section{ACKNOWLEDGMENTS}

This work is supported by TÜBİTAK 1001 programme, grant no 107E137. The authors gratefully acknowledge the facilities of the Unmanned Air Vehicles Laboratory of TOBB Economics and Technology University.

\section{REFERENCES}

Chu, S.R. Shoureshi, R. Tenorio, M. (1990). Neural Networks for System Identification. IEEE Control Systems Magazine. 10, 31-35.

Haykin, S. (1994). Neural Networks, Macmillan College Printing Company, New Jersey.

Hagan, M. and Menhaj, M. (1994). Training Feedforward Networks with the Marquardt Algorithm. IEEE Transactions on Neural Networks, 5(6):989-993.

Juang J.-N. (1994). Applied System Identification, Prentice Hall.

Kim, I.-H., Fok, S., Fregene, K., Lee, D.H., Oh, T.S. and Wang DWL, (2004). Neural Network-Based System
Identification and Controller Synthesis for an Industrial Sewing Machine. Int. Journal of Control Automation and Systems, 2, 83-91.

Ljung, L. (1999). System Identification: Theory for the User, Prentice Hall.

Mohamed, F.A. and Koivo, H. (2004). Modelling of Induction Motor Using Non-Linear Neural Network System Identification. SICE 2004 Annual Conference 2 977-982.

Narendra, K.S. and Parthasarthy, K. (1990). Identification and Control of Dynamical Systems Using Neural Networks. IEEE Trans. on Neural Networks. 1, 4-27.

Negnevitsky, M., and Pavlovsky, V. (2005). Neural Networks Approach to Online Identification of Multiple Failures of Protection Systems. IEEE Trans. on Power Delivery, 588-594.

Pounds, P., Mahony, R. and Corke, P. (2006). Modelling and Control of a Quad-Rotor Robot. Proc. of the Australasian Conference on Robotics and Automation, December 6 8, Auckland, New Zealand.

Rubaai, A., Kotaru, R. and Kankam, M.D. (1997). A RealTime Neural Network Based Controller for Brushless DC Motor Drives. 1997 IEEE Industry Applications Conference, IAS'97, October 5-9, 2, 828-835, New Orleans, LA, USA.

Tipsuwanporn, V., Piyarat, W. and Tarasantisuk, C. (2002). Identification and Control of Brushless DC Motors Using On-line Trained Artificial Neural Networks. Proc. of the Power Conversion Conference PCC'02. 3, 1290 1294. 